\title{
Impact of the peripartum period on the longitudinal course of obsessive-compulsive disorder
}

\author{
Aparna Chatterjee $e^{1,2}\left(\right.$ D $\cdot$ Katelyn Zumpf ${ }^{3} \cdot$ Jennifer Sprague ${ }^{1,4} \cdot$ Jody Ciolino $^{3} \cdot$ Katherine L. Wisner $^{1} \cdot$ Crystal Clark $^{1}$. \\ Maria C. Mancebo ${ }^{5,6}$. Jane L. Eisen ${ }^{7,8}$. Steven A. Rasmussen ${ }^{5}$. Christina L. Boisseau ${ }^{1}$
}

Received: 28 September 2020 / Accepted: 13 April 2021 / Published online: 21 April 2021

(c) The Author(s), under exclusive licence to Springer-Verlag GmbH Austria, part of Springer Nature 2021

\begin{abstract}
Some women are vulnerable to developing new onset obsessive-compulsive disorder (OCD) or having an exacerbation of pre-existing OCD during reproductive cycle events. Reports on the impact of the peripartum period on pre-existing OCD are inconsistent, with both worsening and improving symptom severity described. Studies have primarily been retrospective or have collected few data points, which limits the investigators' ability to capture the range of OCD symptoms during this time period, systematically and prospectively. The objective of this investigation was to add to the existing literature on the impact of the peripartum period on the course of pre-existing OCD. We conducted a secondary analysis of a subset data from the Brown Longitudinal Obsessive Compulsive Study, a prospective, observational study of OCD course. Nineteen women who experienced a pregnancy during the course of the study $(9.5 \%$ of overall sample of women) were followed on average for $486 \pm 133$ weeks. Weekly psychiatric status ratings (PSRs) of OCD severity were compared between peripartum and non-peripartum periods. We found that the peripartum period did not significantly impact the course of OCD severity in the majority of women $(N=13,69 \%)$. Of the minority of women with measurable variability in OCD symptoms, no statistically significant difference in PSR scores was observed between peripartum and non-peripartum periods. In this novel yet small dataset, the severity of OCD does not appear to worsen for most women during the peripartum period.
\end{abstract}

Keywords Reproductive cycle events · Obsessive-compulsive disorder · Pregnancy · Peripartum · Prospective study

Women's mental health during the Covid-19 pandemic

Edited by: Anita Riecher-Rössler

Aparna Chatterjee

apchatter@gmail.com

1 Department of Psychiatry and Behavioral Sciences, Northwestern University Feinberg School of Medicine, Chicago, IL, USA

2 Meridian Psychiatric Partners, Chicago, IL, USA

3 Department of Preventive Medicine, Division of Biostatistics, Northwestern University Feinberg School of Medicine, Chicago, IL, USA

4 Advocate Illinois Masonic Medical Center, Chicago, IL, USA

5 Department of Psychiatry and Human Behavior, Warren Alpert Medical School of Brown University, Chicago, IL, USA

6 Butler Hospital, Providence, RI, USA

7 Department of Psychiatry, Harvard Medical School, Boston, MA, USA

8 McLean Hospital, Belmont, MA, USA

\section{Introduction}

Pregnancy and postpartum have been shown to be a high-risk period for onset or exacerbation of psychiatric illness (O'Hara and Wisner 2014). Much of the literature has focused on mood disorders during this time, but there is less information evaluating the relationship between obsessive-compulsive disorder (OCD) and the perinatal period (Abramowitz et al. 2003). The available data indicates that reproductive cycle events including menarche, pregnancy, and the postpartum period have been shown to be a high-risk period for new onset OCD in women (Forray et al. 2010; Guglielmi et al. 2014; Labad et al. 2005). OCD during pregnancy can have a significant negative impact on physical and psychological health, as well as social relationships, in which disease severity is negatively correlated with quality of life (Gezginc et al. 2008). In one study, mothers with OCD reported decreased self-efficacy and increased marital distress during the postpartum period (Challacombe et al. 2016). They were also less likely to breastfeed and 
reported more difficulties with attending to the care of their babies (Challacombe et al. 2016). In addition to the negative impact on mothers, OCD during pregnancy has been associated with unfavorable outcomes in the neonate, including lower birth weight and shorter gestation (Uguz et al. 2015). Given these potential negative outcomes to both mother and infant, it is important to clarify the impact of the peripartum period on severity and symptom course of OCD.

While investigators have focused on reproductive cycle events as a high-risk time for OCD onset, the impact of pregnancy on pre-existing OCD is equivocal with reports showing improvement in 8-28\% (Guglielmi et al. 2014), worsening in 17-46\% (Uguz et al. 2007; Williams and Koran 1997), and no change in 31-69\% (Uguz et al. 2007; Williams and Koran 1997). Report of the postpartum period is similar; most women report no change or exacerbation of OCD symptoms (Guglielmi et al. 2014; Labad et al. 2005; Williams and Koran 1997), albeit a smaller percentage of women $(0-10 \%)$ report symptom improvement (Guglielmi et al. 2014; Labad et al. 2005). Most data on perinatal OCD symptoms are obtained from retrospective studies which are subject to recall bias and single point, cross-sectional data, which limit ability to track OCD trajectory throughout the peripartum period (Forray et al. 2010; Guglielmi et al. 2014; Labad et al. 2005; Uguz et al. 2007; Williams and Koran 1997).

The only published prospective study following women with obsessive-compulsive symptoms (OCS) during pregnancy and the postpartum period found no change in OCS severity during the perinatal period (House et al. 2016). This study included 52 participants whose symptoms were monitored across pregnancy and 1 year postpartum with the Yale-Brown Obsessive Compulsive Scale (YBOCS). A minimum of three data points were obtained with at least one assessment in pregnancy and one in postpartum. The authors concluded that OCS did not change over the course of pregnancy and postpartum. This study only obtained a few data points and primarily included women with mild or subclinical presentations which again limited the investigators' ability to capture OCD variability and trajectory.

The objective of this investigation was to determine the impact of the peripartum period on the course of pre-existing OCD. Utilizing weekly data from a prospective longitudinal study of OCD course, we sought to explore individual trajectories of OCD severity during the peripartum period and compare symptom course between peripartum and nonperipartum states. Based on the study conducted by House and colleagues (House et al. 2016), we hypothesized a waxing and waning course of symptoms would be observed, but overall OCD course would not be significantly affected by the peripartum period.

\section{Materials and methods}

\section{Participants}

We conducted a secondary analysis of a subset of data from women who experienced a pregnancy while participating in the Brown Longitudinal Obsessive Compulsive Study (BLOCS), a prospective, observational study of OCD course with 200 females (177 adults, 23 adolescents). A detailed description of the sample characteristics and study methodology has been reported elsewhere (Pinto et al. 2006). Briefly, treatment-seeking participants with a primary psychiatric diagnosis (primary psychiatric disorder across their lifetime) of OCD as defined by the Diagnostic and Statistical Manual of Mental Disorders, Fourth Edition (DSM-IV) were recruited from inpatient, partial hospital, and outpatient settings from 2001 to 2006 . The only exclusion criterion was the presence of a mental disorder due to a medical condition. All participants consented to having their data collected, analyzed, and used in publication. The study was approved by the institutional review board at Butler Hospital and Brown University.

\section{Measures}

The Structured Clinical Interview for DSM-IV Axis I Disorders-Patient Edition ${ }^{1}$ was used to establish Axis I diagnoses at intake (First et al. 2002). Demographic and clinical history were assessed using the Butler Hospital OCD Database (Rasmussen 1993). The YBOCS was used to assess OCD symptoms and severity at the initial assessment (Goodman et al. 1989a, b). The YBOCS is widely considered the gold standard instrument for OCD symptom assessment and has well-established reliability and validity (Goodman et al. 1989a, b). Psychiatric diagnoses and YBOCS total scores in this study have been found to have good to excellent interrater reliability as reported elsewhere (Eisen et al. 2010). Medication inventory was taken at baseline.

Participants underwent annual follow-up interviews either in person or by telephone. This assessment included the Longitudinal Interval Follow-Up Evaluation (LIFE), a semi-structured instrument designed to assess the longitudinal course of psychiatric disorders (Keller et al. 1987). Using information obtained through the LIFE, weekly psychiatric status ratings (PSRs) were made to determine whether participants met diagnostic criteria for specific psychiatric disorders. The 6-point OCD PSR indicated

\footnotetext{
${ }^{1}$ One participant entered this study at the age of 14 and became pregnant as an adult during the follow-up period. Intake diagnoses for this participant were made using the Kiddie Schedule for Affective Disorders and Schizophrenia (Kaufman et al. 1997) and the child version of the YBOCS (Scahill et al. 1997).
} 
whether participants met criteria for OCD on a range of severity, distress, and impairment. This range included no symptoms (PSR 1), minimal symptoms (PSR 2), mild symptoms (PSR 3), moderate symptoms (PSR 4), severe symptoms (PSR 5), or extreme symptoms (PSR 6). A PSR of $\geq 4$ indicates full criteria are met for the disorder. Psychiatric status ratings are frequently used in longitudinal studies and provide reliable and valid ratings of ongoing disorder severity (Warshaw et al. 1994). Inter-rater and test-retest reliability for OCD PSR ratings in this sample are excellent and have been reported elsewhere (Eisen et al. 2010), as have our methods for establishing and maintaining long-term reliability (Pinto et al. 2006).

Life events were assessed with Life Event Assessment (LEA) which captured the month and year for each pregnancy occurring in each follow-up interval (Dohrenwend et al. 1978). We used an algorithm to estimate the peripartum period. The algorithm defined the start of the peripartum period as 4 weeks prior to the first day of the month in which the pregnancy was documented. We defined the end of the peripartum window as 12 weeks after the first day of the month in which a birth or miscarriage occurred. This time window was chosen to demarcate the peripartum period in line with the theory of four trimesters of pregnancy (Tully et al. 2017). Additionally, this period is reflected in the DSM-5 which defines "with peripartum onset" as an episode occurring during pregnancy as well as 4 weeks postpartum. We extended our postpartum period to 12 weeks as this is consistent with epidemiological data that found elevated rates of psychiatric contacts through the first 3 months after childbirth (Kendell et al. 1987; Munk-Olsen et al. 2006), and proposals that the DSM-5 peripartum specifier does not adequately describe at-risk periods across moderate and severe perinatal psychiatric episodes (Munk-Olsen et al. 2016).

When a birth occurred without a pregnancy indicated in the database, we defined the start of the peripartum period as 44 weeks prior to the first day of the month in which the birth occurred. Similarly, if a miscarriage occurred without knowledge of a pregnancy, the start of peripartum period was set as 12 weeks prior to the first of the month. Thus, the algorithm provided peripartum epochs that varied within and between individuals, depending upon documentation within the database.

Detailed descriptions of rater training are reported in Pinto et al. (2006). Briefly, all interviewers had at least a bachelor's degree and were supervised by MD- and PhDlevel experts in OCD research and treatment. Interviewers completed a series of observational, didactic, and participatory training activities including conducting assessment with feedback from experienced interviewers and audiotaping for inter-rater reliability calculations. New interviewers were required to achieve excellent inter-rater reliability with trainers (interclass correlation coefficient $>0.85$ ) for the SCID, YBOCS total score, and the LIFE. Through the study, fidelity to training was monitored via weekly meetings to review ratings as well as periodic, random calculation of inter-rater reliability statistics.

\section{Statistical analysis}

Our analyses focused on the primary outcome of OCD PSR (range: 1-6). We created individual participant profile plots to illustrate longitudinal symptom course over the observed period. The original analysis plan called for a mixed-effects or a generalized estimating equation model to determine whether OCD PSR scores changed during the peripartum period. We planned to account for within-participant correlation through either random effects or appropriate covariance assumptions. However, since all participants entered the study with a PSR $\geq 3$, we observed little variability in outcome over time. This resulted in challenges with model convergence, which was true whether we modeled outcome as continuous, ordinal, or dichotomous based on PSR $\geq 4$. Therefore, we simplified analyses and collapsed data for each participant on peripartum and non-peripartum states. We calculated an average OCD PSR score for each participant during these periods and used a Wilcoxon signed-rank test to evaluate differences in mean OCD PSR scores between peripartum and non-peripartum states. For participants with measurable variability, we constructed individual logistic regression models for OCD PSR $\geq 4$. The models contained a peripartum-by-week interaction and corresponding main effects. Individual estimates for the change in log-odds of OCD PSR $\geq 4$ for 1-week increase in time (i.e., the interaction between peripartum indicator and week) were captured and summarized using a random effects meta-analysis with the restricted maximum-likelihood estimator (REML) for between- "study" (i.e., between participant) variance, $\tau^{2}$. We performed analyses using R (3.5.3, 2019, The R Foundation) and assumed a two-sided, 5\% level of significance. Metaanalysis was conducted using the metaphor package (Viechtbauer 2010). Since this was a secondary data analysis, no formal power and sample size calculation was performed.

\section{Results}

We identified 19 women $(9.5 \%$ of women in the BLOCS cohort) who had a pregnancy, birth, or miscarriage documented in the database. Just more than half of the women $(N=10,52.6 \%)$ reported more than one pregnancy through the course of follow-up. The average length of follow-up was $486 \pm 133$ weeks, ranging from 208 to 676 weeks. Participant demographics, medication use, and psychiatric history were obtained at study intake. The mean age was 25.9 years ( $\mathrm{SD}=6.2$; range of $14-38$ years) with the majority being white $(94.7 \%)$, employed (63.2\%), with some college or 
graduate school education (89.5\%), and no reported disability. The majority of women $(73.7 \%)$ reported a history of comorbid major depressive disorder. Additional comorbidities included history of an anxiety disorder (36.8\%), eating disorder $(21.1 \%)$, tic disorder (15.8\%), or substance abuse (5.3\%). Most participants $(N=16,84.2 \%)$ were prescribed a serotonin reuptake inhibitor. Additionally, 2 participants were treated with a neuroleptic and 3 participants were treated with a benzodiazepine.

At intake, participants reported a moderate degree of OCD severity with a mean YBOCS score of $18.9(\mathrm{SD}=8.2)$. Symmetry or exactness was the most common obsession (26.3\%) followed by a fear of harming others (15.8\%) (Fig. 1). At study entry, all women scored between 3 and 5 points on the PSR, representing a range of symptom severity from mild to severe, with the majority (52.6\%) scoring a PSR of 4, representing moderate symptom presentation (Table 1). No evidence of a statistically significant difference in participant average PSR scores was observed between peripartum and non-peripartum states (paired [pseudo] median difference: -0.0082 ; 95\% CI: $[-0.25,0.18]$; Wilcoxon rank-sum $p$-value $=0.90$ ).

The symptom PSR scores varied little within participants overall, where subject 7 had the same score for over 300 weeks (Fig. 1). Scores remained constant during the peripartum period for seven participants $(1,7,9,13,15,16$, and 19). Six participants $(2,3,5,8,14$, and 18$)$ exhibited enough variability in PSR to model PSR scores over time. We conducted the aforementioned meta-analysis using these participants' data (Fig. 2). Despite the fact that 3 of the 6 participants $(2,3,5)$ had a significant 2-way interaction between peripartum state and time, overall there was insufficient evidence to suggest that the association between time and OCD PSR $\geq 4$ differed between peripartum
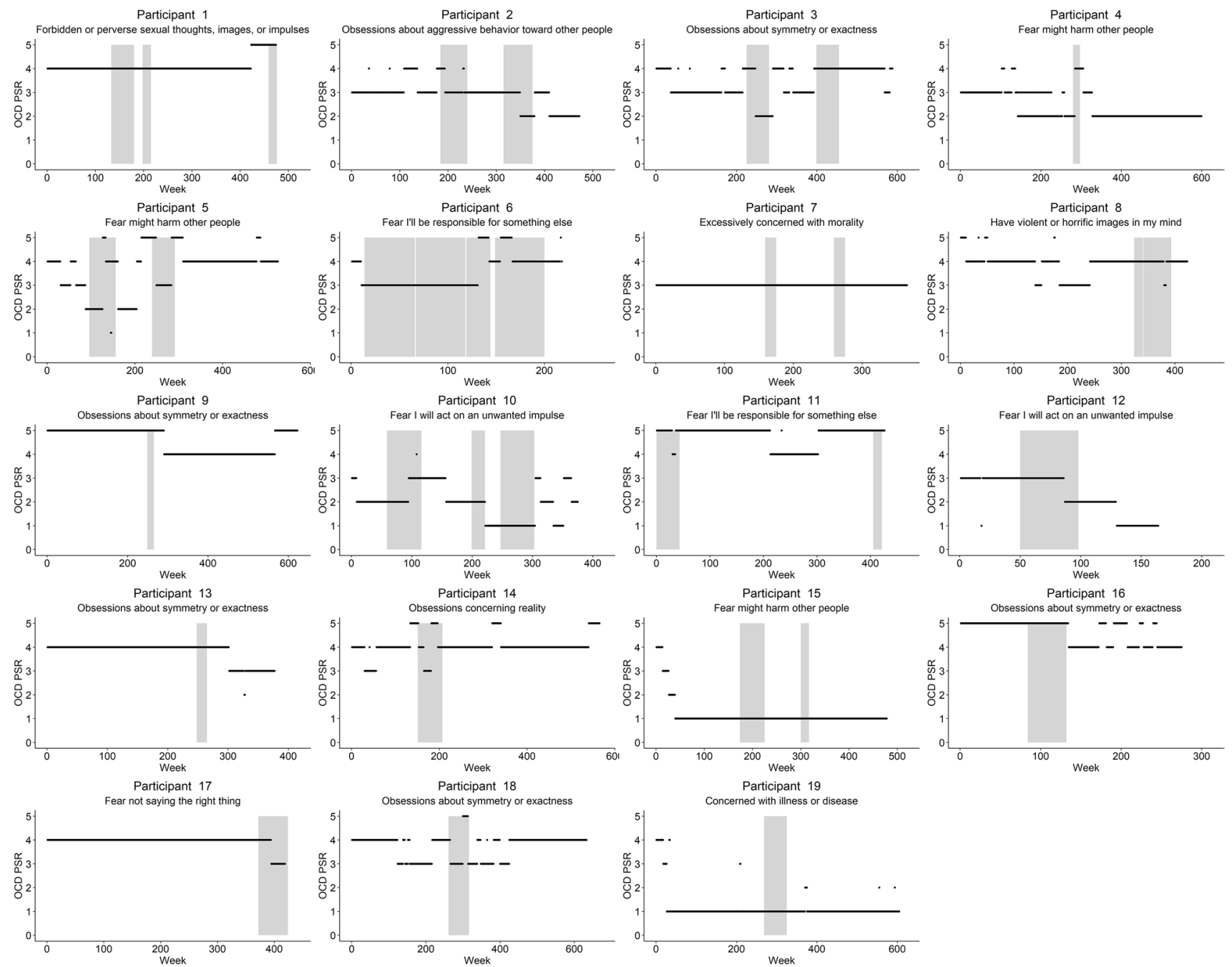

Fig. 1 Distribution of obsessive-compulsive disorder psychiatric status rating (OCD PSR) scores over time and stratified by peripartum state. A 6-point OCD PSR was used to indicate a range of OCD severity where PSR 1 indicated no symptoms and PSR 6 indicated extreme symptoms. These graphs depict all nineteen participant's

OCD trajectories via weekly PSR scores, represented as black horizontal dots. These PSR scores are graphed spanning peripartum and non-peripartum periods, where peripartum time windows are depicted with a vertical gray box. The participant's predominant obsession is also documented with each graph 
Table 1 Summary of baseline obsessive-compulsive disorder psychiatric status rating $(\mathrm{OCD}$ PSR) scores in the sample

\begin{tabular}{ll}
\hline OCD PSR & $\begin{array}{l}\text { Overall } \\
(n=19)\end{array}$ \\
\hline 1 & $0(0 \%)$ \\
2 & $0(0 \%)$ \\
3 & $5(26.3 \%)$ \\
4 & $10(52.6 \%)$ \\
5 & $4(21.1 \%)$ \\
6 & $0(0 \%)$ \\
\hline
\end{tabular}

OCD PSR scores indicate level of severity of OCD with higher scores representing more severe symptom presentation

and non-peripartum states for these women $(n=6$; overall odds ratio for OCD PSR $\geq 4: 0.99 ; 95 \%$ confidence interval: $(0.96$, $1.02) ; p$-value $=0.57)$.

\section{Discussion}

The peripartum period does not appear to be associated with a worsening of OCD symptom severity for most women in our study. Throughout the peripartum period, symptoms were unchanged for the majority (69\%) of participants. A subset of patients $(31.6 \%)$ reported variability with improvement or worsening of symptoms; however, the change in overall symptom severity during the individual woman's peripartum state compared to her non-peripartum state was not clinically significant. Consistent with prior investigations (Forray et al. 2010; Guglielmi et al. 2014; House et al. 2016; Labad et al. 2005; Williams and Koran 1997), we found that

the peripartum period did not exacerbate pre-existing OCD for most women. However, in one retrospective study (Uguz et al. 2007), a slightly higher proportion of women experienced worsening or improvement of symptoms (46.1\% and $23.1 \%$, respectively) compared to women with no change (30.8\%). This difference in findings may be explained by cross-sectional data collection in the third trimester, which limits the investigators' ability to assess the impact of the full course of the peripartum period on OCD trajectory. Broader longitudinal follow-up studies of this sample underscore the chronicity of OCD (Eisen et al. 2013) and our findings, and the majority of previous studies, suggest that this may also be true for OCD during the peripartum period.

The strengths of this prospective study include a wellcharacterized sample with a long period of observation. Weekly PSR data through peripartum and non-peripartum periods allowed us to examine the impact of the peripartum period on overall trajectory of OCD. Our participants had a moderate level of symptom severity, which expands upon past research that included women with mild or subclinical presentations (House et al. 2016).

Although there were a large number of observations per participant, the primary limitation of this study is the small sample size and limited variability in OCD PSR. These restricted the statistical methods that could be performed. In addition, inclusion of multiple events (pregnancy, birth, and miscarriage) as one category assumes that they have the same impact on OCD symptoms. Psychiatric treatment (medication, psychotherapy) was uncontrolled so we are not able to draw conclusions about its effects on our analyses. Finally, the sample was predominantly white and all participants were treatment seeking, which limits the generalizability of these results.

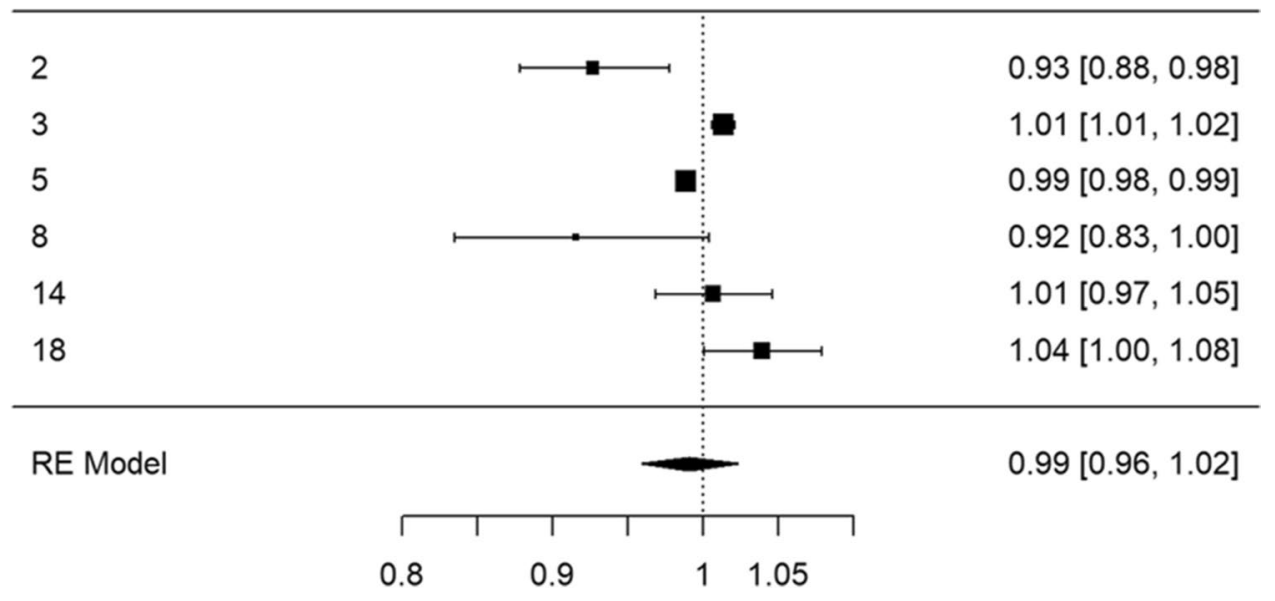

Fig. 2 Meta-analysis of subjects with higher variability in obsessive-compulsive disorder psychiatric status rating (OCD PSR) scores in the peripartum. Of the nineteen participants in this study, six participants demonstrated higher variability in OCD PSR scores during the peripartum. OCD PSR scores represent a marker of OCD severity, with higher numbers indicating a greater severity. The OCD PSR scores were dichotomized to $\mathrm{PSR}<4$ and $\mathrm{PSR} \geq 4$, where $\mathrm{PSR} \geq 4$ indicates full criteria is met for the disorder. An odds ratio and $95 \%$ confidence interval were calculated to determine if there was a change between these two dichotomized PSR scores across peripartum and non-peripartum states 
Despite these limitations, our observations comprise realworld experiences in a population of women with OCD in long-term follow-up. We provide new data that the severity of OCD does not appear to be impacted by the peripartum period. Additionally, we show that while the peripartum period does not typically impact the overall trajectory of OCD, there is potential for symptom variability in some women. This demonstrates a heterogeneity of response with either improvement or worsening of symptoms consistent with past literature (Forray et al. 2010; Guglielmi et al. 2014; House et al. 2016; Labad et al. 2005; Uguz et al. 2007; Williams and Koran 1997). Future studies should distinguish obsessions/compulsions in pregnancy from postpartum as research suggests certain obsessions like symmetry may be associated with higher disease severity and comorbidity (Sibrava et al. 2016) and the postpartum period may be a higher risk time for OCD exacerbation (Guglielmi et al. 2014; Labad et al. 2005).

\section{Conclusion}

In conclusion, OCD severity does not appear to change across the peripartum period for the majority of women in our study. This study adds to our knowledge of the longitudinal impact of perinatal events on OCD symptomology and provides clinicians with considerations as to how to counsel and monitor patients with OCD during the peripartum.

Author contribution $\mathrm{CB}, \mathrm{KW}, \mathrm{CC}, \mathrm{JC}$, and $\mathrm{JS}$ contributed to the concept and design of the paper. CB, SR, MM, JE, KZ, JC, CC, and $\mathrm{KW}$ and contributed to the acquisition, analysis, and interpretation of data. KW and JC contributed to statistical analysis. AC and JS drafted the manuscript. All authors contributed to the critical revision of the manuscript for important intellectual content and approved the final manuscript.

Funding This study was funded by a grant from the National Institutes of Health [R01 MH060218]. The funding source had no involvement in the study design; the collection, analysis, and interpretation of the data; in the writing of the report; and in the decision to submit the article for publication.

\section{Declarations}

Ethics approval All procedures performed in studies involving human participants were in accordance with the ethical standards of the institutional and/or national research committee and with the 1964 Helsinki declaration and its later amendments or comparable ethical standards. This study received IRB approval and informed consent was obtained from all individual participants included in the study.

Conflict of interest The authors declare no competing interests.

\section{References}

Abramowitz JS, Schwartz SA, Moore KM, Luenzmann KR (2003) Obsessive-compulsive symptoms in pregnancy and the puerperium: a review of the literature. J Anxiety Disord 17:461-478. https://doi.org/10.1016/s0887-6185(02)00206-2

Challacombe FL, Salkovskis PM, Woolgar M, Wilkinson EL, Read J, Acheson R (2016) Parenting and mother-infant interactions in the context of maternal postpartum obsessive-compulsive disorder: effects of obsessional symptoms and mood. Infant Behav Dev 44:11-20. https://doi.org/10.1016/j.infbeh.2016.04.003

Dohrenwend BS, Krasnoff L, Askenasy AR, Dohrenwend BP (1978) Exemplification of a method for scaling life events: the Peri Life Events Scale. J Health Soc Behav 19:205-229

Eisen JL, Pinto A, Mancebo MC, Dyck IR, Orlando ME, Rasmussen SA (2010) A 2-year prospective follow-up study of the course of obsessive-compulsive disorder. J Clin Psychiatry 71:10331039. https://doi.org/10.4088/JCP.08m04806blu

Eisen JL, Sibrava NJ, Boisseau CL, Mancebo MC, Stout RL, Pinto A, Rasmussen SA (2013) Five-year course of obsessive-compulsive disorder: predictors of remission and relapse. J Clin Psychiatry 74:233-239. https://doi.org/10.4088/JCP.12m07657

First M, Spitzer R, Gibbon M et al (2002) Structured clinical interview for DSM-IV axis I disorders - patient edition (SCID-I/P, Version 2.0). Biometrics Research, New York State Psychiatric Institute, New York

Forray A, Focseneanu M, Pittman B, McDougle CJ, Epperson CN (2010) Onset and exacerbation of obsessive-compulsive disorder in pregnancy and the postpartum period. J Clin Psychiatry 71:1061-1068. https://doi.org/10.4088/JCP.09m05381blu

Gezginc $\mathrm{K}$ et al (2008) The impact of obsessive-compulsive disorder in pregnancy on quality of life. Int J Psychiatry Clin Pract 12:134-137. https://doi.org/10.1080/13651500701777363

Goodman WK, Price LH, Rasmussen SA, Mazure C, Delgado P, Heninger GR, Charney DS (1989a) The Yale-Brown Obsessive Compulsive Scale II Validity. Arch Gen Psychiatry 46:1012-1016. https://doi.org/10.1001/archpsyc.1989.01810110054008

Goodman WK et al (1989b) The Yale-Brown Obsessive Compulsive Scale. I Development, use, and reliability. Arch Gen Psychiatry 46:1006-1011. https://doi.org/10.1001/archpsyc.1989.01810 110048007

Guglielmi V, Vulink NC, Denys D, Wang Y, Samuels JF, Nestadt G (2014) Obsessive-compulsive disorder and female reproductive cycle events: results from the OCD and reproduction collaborative study. Depress Anxiety 31:979-987. https://doi.org/10.1002/da.22234

House SJ, Tripathi SP, Knight BT, Morris N, Newport DJ, Stowe ZN (2016) Obsessive-compulsive disorder in pregnancy and the postpartum period: course of illness and obstetrical outcome. Arch Women's Mental Health 19:3-10. https://doi.org/10.1007/ s00737-015-0542-Z

Kaufman J et al (1997) Schedule for Affective Disorders and Schizophrenia for School-Age Children-Present and Lifetime Version (K-SADS-PL): initial reliability and validity data. J Am Acad Child Adolesc Psychiatry 36:980-988. https://doi.org/10.1097/ 00004583-199707000-00021

Keller MB, Lavori PW, Friedman B, Nielsen E, Endicott J, McDonaldScott P, Andreasen NC (1987) The longitudinal interval follow-up evaluation. A comprehensive method for assessing outcome in prospective longitudinal studies. Arch Gen Psychiatry 44:540 548. https://doi.org/10.1001/archpsyc.1987.01800180050009

Kendell RE, Chalmers JC, Platz C (1987) Epidemiology of puerperal psychoses. Br J Psychiatry 150:662-673. https://doi.org/10.1192/ bjp. 150.5 .662 
Labad J, Menchon JM, Alonso P, Segalas C, Jimenez S, Vallejo J (2005) Female reproductive cycle and obsessive-compulsive disorder. J Clin Psychiatry 66:428-435; quiz 546 https://doi.org/10.4088/jcp.v66n0404

Munk-Olsen T, Laursen TM, Pedersen CB, Mors O, Mortensen PB (2006) New parents and mental disorders a population-based register study. JAMA 296:2582-2589. https://doi.org/10.1001/jama.296.21.2582

Munk-Olsen T et al (2016) Perinatal psychiatric episodes: a populationbased study on treatment incidence and prevalence. Transl Psychiatry 6:e919. https://doi.org/10.1038/tp.2016.190

O'Hara MW, Wisner KL (2014) Perinatal mental illness: definition, description and aetiology. Best Pract Res Clin Obstet Gynaecol 28:3-12. https://doi.org/10.1016/j.bpobgyn.2013.09.002

Pinto A, Mancebo MC, Eisen JL, Pagano ME, Rasmussen SA (2006) The Brown Longitudinal Obsessive Compulsive Study: clinical features and symptoms of the sample at intake. J Clin Psychiatry 67:703-711. https://doi.org/10.4088/jcp.v67n0503

Rasmussen SA (1993) Genetic studies of obsessive-compulsive disorder. Ann Clin Psychiatry 5:241-247. https://doi.org/10.3109/ 10401239309148823

Scahill L et al (1997) Children's Yale-Brown Obsessive Compulsive Scale: reliability and validity. J Am Acad Child Adolesc Psychiatry 36:844 852. https://doi.org/10.1097/00004583-199706000-00023

Sibrava NJ, Boisseau CL, Eisen JL, Mancebo MC, Rasmussen SA (2016) An empirical investigation of incompleteness in a large clinical sample of obsessive compulsive disorder. J Anxiety Disord 42:45-51. https://doi.org/10.1016/j.janxdis.2016.05.005
Tully KP, Stuebe AM, Verbiest SB (2017) The fourth trimester: a critical transition period with unmet maternal health needs. Am J Obstet Gynecol 217:37-41. https://doi.org/10.1016/j.ajog.2017. 03.032

Uguz F et al (2007) Obsessive-compulsive disorder in pregnant women during the third trimester of pregnancy. Compr Psychiatry 48:441-445. https://doi.org/10.1016/j.comppsych.2007.05.001

Uguz F, Yuksel G, Karsidag C, Guncu H, Konak M (2015) Birth weight and gestational age in newborns exposed to maternal obsessivecompulsive disorder. Psychiatry Res 226:396-398. https://doi.org/ 10.1016/j.psychres.2014.12.063

Viechtbauer W (2010) Conducting meta-analyses in R with the metafor Package 36:48. https://doi.org/10.18637/jss.v036.i03

Warshaw MG, Keller MB, Stout RL (1994) Reliability and validity of the longitudinal interval follow-up evaluation for assessing outcome of anxiety disorders. J Psychiatr Res 28:531-545. https:// doi.org/10.1016/0022-3956(94)90043-4

Williams KE, Koran LM (1997) Obsessive-compulsive disorder in pregnancy, the puerperium, and the premenstruum. J Clin Psychiatry 58:330-334; quiz 335-336. https://doi.org/10.4088/jcp. v58n0709

Publisher's note Springer Nature remains neutral with regard to jurisdictional claims in published maps and institutional affiliations. 